\title{
Vitamin D and Gestational Diabetes Mellitus
}

\author{
Heather H. Burris, MD, MPH and \\ Department of Neonatology ${ }^{1}$, Beth Israel Deaconess Medical Center, and Division of Newborn \\ Medicine, Boston Children's Hospital and Harvard Medical School.
}

Carlos A. Camargo Jr., MD, DrPH

Department of Emergency Medicine, Massachusetts General Hospital, and Channing Division of Network Medicine, Department of Medicine, Brigham and Women's Hospital, Harvard Medical School, Boston, MA, USA.

Carlos A. Camargo: ccamargo@partners.org

\begin{abstract}
Gestational diabetes mellitus (GDM) complicates $7-14 \%$ of pregnancies in the United States. Vitamin D deficiency also is common in pregnancy. Emerging evidence suggests that Vitamin D administration can improve insulin sensitivity and glucose tolerance, but whether vitamin D supplementation can prevent GDM is unknown. Observational studies provide conflicting evidence as to whether low serum 25-hydroxyvitmain $\mathrm{D}(25(\mathrm{OH}) \mathrm{D})$ levels are associated with GDM. Two recent systematic reviews concluded that vitamin D deficiency is associated with a higher risk of GDM. However, these reviews are limited by the observational and diverse nature of the included studies. Of greatest concern is the inability to understand how important confounding variables such as race/ethnicity and adiposity might affect the association. Randomized controlled trial data remain limited but are critical to understanding whether supplementation with vitamin $\mathrm{D}$ beyond what is contained in routine prenatal vitamins will prevent GDM or improve glucose tolerance for women with GDM.
\end{abstract}

\section{Keywords}

Vitamin D; 25-hydroxyvitamin D; pregnancy; gestational diabetes mellitus; GDM; gestational diabetes

\section{Introduction}

Immense interest persists in vitamin D and its potential effects on several pregnancy outcomes including fetal growth, hypertensive disorders and gestational diabetes mellitus (GDM). Two factors make vitamin D intriguing to perinatal investigators studying GDM. First, vitamin $\mathrm{D}$ has been shown to improve pancreatic exocrine function and insulin sensitivity in animal models. Second, vitamin D status, like most micronutrients, is easily modified by dietary supplementation. If shown to prevent or improve outcomes of

Corresponding Author: ${ }^{1} 330$ Brookline Avenue, RO 318, Boston, MA 02215. Phone (617) 667-3276. Fax (617) 667-667-7040. heburris@bidmc.harvard.edu.

Compliance with Ethics Guidelines

Conflict of Interest

Heather H. Burris and Carlos A. Camargo, Jr. declare that they have no conflict of interest.

Human and Animal Rights and Informed Consent

This article does not contain any studies with human or animal subjects performed by any of the authors. 
pregnancies complicated by GDM, vitamin D intake could be titrated to achieve optimal serum 25-hydroxyvitamin D (25(OH)D) levels.

To date, the literature does not support routine high-dose vitamin D supplementation during pregnancy for either the prevention or the treatment of GDM. In this review, we will briefly describe the metabolic functions of vitamin D and the epidemiology of GDM. We will present the most recent observational studies linking vitamin D to GDM, including results from systematic reviews and meta-analyses, and results from the few interventional trials to date. We will highlight the challenges faced when reading these diverse studies and propose a future research agenda to investigate whether GDM or its complications could be either prevented or mitigated by optimal vitamin D status.

\section{Vitamin D}

Vitamin D, also known as calciferol, includes two major, functionally identical forms, vitamin D2 (ergocalciferol) which is synthesized and added to foods and supplements, and vitamin D3 (cholecalciferol) which is present in animal-based foods and made by human skin through a sunlight-induced conversion of 7-dehydrocholesterol [1]. Both forms are prohormones, and inactive until hydroxylated twice: first in the liver to form 25hydroxyvitamin $\mathrm{D}(25[\mathrm{OH}] \mathrm{D})$, and then again in the kidney to form the biologically active hormone, calcitriol (1,25-dihydroxyvitamin D). The major circulating form of vitamin $\mathrm{D}$ is $25(\mathrm{OH}) \mathrm{D}$ which is bound in plasma to vitamin $\mathrm{D}$ binding protein (DBP) and albumin, and is the best available marker of overall vitamin D status. Calcitriol synthesis in the kidney is tightly regulated by parathyroid hormone. Calcitriol regulates gene expression by affecting gene transcription through interaction with a nuclear vitamin D receptor (VDR). The traditional role of calcitriol is to regulate serum calcium and phosphate homeostasis and thus maintain bone health.

However, VDRs are found in tissues that are not directly involved in calcium or phosphate metabolism suggesting that calcitriol might have functions beyond its traditional role in bone health [1]. Vitamin D-responsive elements (VDRE) are present in several human genes involved in cell differentiation and proliferation and thus vitamin $\mathrm{D}$ has been studied as a potential therapeutic or preventative candidate for cancer [2] and autoimmune diseases including type 1 diabetes mellitus [3]. In rodent models, calcitriol has been shown to have effects on the synthesis, secretion and actions of insulin $[4,5]$, leading to several human observational and interventional studies of vitamin D and type 2 diabetes mellitus, a few of which have shown a potential benefit of vitamin D supplementation or optimal $25(\mathrm{OH}) \mathrm{D}$ levels on type 2 diabetes [6]. Such studies have prompted a growing number of studies on the relationship between vitamin D status and GDM.

\section{Gestational Diabetes Mellitus (GDM)}

The increasing rates of overweight and obesity in the general population are undoubtedly contributing to the ongoing rise in the prevalence of GDM [7], which now complicates approximately $7-14 \%$ of pregnancies in the United States [8, 9]. GDM places both mothers and their infants at risk for adverse health consequences [10]. Women with GDM are more likely to undergo cesarean section and later develop type 2 diabetes mellitus. Infants of diabetic mothers are more likely to have congenital anomalies, macrosomia, birth trauma, respiratory distress syndrome, jaundice and hypoglycemia. While several GDM risk factors have been identified [11] - including advanced maternal age, obesity, family history of diabetes and ethnicity [12] - how these risk factors predispose women to GDM remains an active area of scientific inquiry [13]. In recent years, vitamin D deficiency has been increasingly recognized as one potential contributor [14]. While epidemiologic studies have shown a fairly consistent link between vitamin D deficiency and a higher risk of type 2 
diabetes [6, 15], and obesity is strongly associated with both GDM [16, 17] and vitamin D deficiency $[2,18,19]$, it remains unclear whether vitamin $\mathrm{D}$ deficiency contributes to a mother's risk of developing GDM.

\section{Observational studies of vitamin D and GDM}

Several, but not all, observational studies have found an association between low 25(OH)D level and increased risk of GDM. In a matched, case-control study of 54 Iranian women with GDM and 11 normoglycemic controls, Soheilykhah et al. found that maternal 25(OH)D concentrations at 24-28 weeks of gestation were significantly lower in women with GDM [20]. They noted that $83 \%$ of GDM women had $25(\mathrm{OH}) \mathrm{D}$ levels $<50 \mathrm{nmol} / \mathrm{L}$ (a cutoff often used to define vitamin D deficiency [21-24]) vs. $71 \%$ of controls. Clifton-Bligh and colleague studied 264 women in Australia and found that among the $32 \%$ with GDM, 25(OH)D levels were significantly lower compared to normoglycemic women [25]. In another study of Iranian women at high risk for vitamin D deficiency, Hossein-Nezhad and colleagues found that $29 \%$ of 741 women had $25(\mathrm{OH}) \mathrm{D}$ levels $<15 \mathrm{nmol} / \mathrm{L}$ and the prevalence of GDM in this subgroup was higher compared to women with $25(\mathrm{OH}) \mathrm{D}$ levels $335 \mathrm{nmol} / \mathrm{L}$ [26]. Likewise, Zhang et al. found in a nested case-control study in the United States (Washington) of 57 cases of GDM, that maternal 25(OH)D levels at 16 weeks' gestation were 20\% lower among women who later developed GDM [27].

However, other studies have not detected a statistically significant association between 25(OH)D level and GDM. Farrant et al studied 559 pregnant women in India and found no association between second trimester 25(OH)D levels and GDM [28]. Likewise, Makgoba and colleagues studied 90 cases of GDM and 158 controls in the United Kingdom and reported no association between first trimester blood samples and subsequent development of GDM [29]. Baker and colleagues conducted a nested case-control study in the United States (North Carolina) using routine first trimester serum aneuploidy screening blood samples, and in their comparison of 60 women who later developed GDM and 120 controls who did not, the investigators found no association between $25(\mathrm{OH}) \mathrm{D}$ level and the odds of GDM.

In addition to skin pigmentation and sun exposure, adiposity and diet can be important determinants of vitamin D status. Physical activity can contribute to sun exposure and reduced adiposity, as well as potentially a decreased risk of GDM. Because none of the above studies adjusted for physical activity or dietary factors, we analyzed data from a pregnancy cohort in Massachusetts that included such variables [30]. Among 1314 pregnant women undergoing routine glucose tolerance screening during pregnancy, we found that women with $25(\mathrm{OH}) \mathrm{D}$ levels $<25 \mathrm{nmol} / \mathrm{L}$ (vs. higher) had higher odds of GDM (OR 3.1, $95 \%$ CI $1.3,7.4$ ) but that this association was attenuated by adjustment for prepregnancy body mass index (OR 2.3, 95\% CI 0.9, 5.7). Further adjustment for physical activity and dietary intakes of fish and calcium did not substantially change the estimate, which remained elevated but was statistically non-significant (OR 2.2, 95\% CI 0.8, 5.5).

Recent systematic reviews (including meta-analyses) have examined the published literature. Wei and colleagues included 12 studies with 5615 participants and concluded that among women with $25(\mathrm{OH}) \mathrm{D}$ levels $<50 \mathrm{nmol} / \mathrm{L}$ there is a modest increase in odds of GDM (crude OR 1.38, 95\% CI 1.12, 1.70) [31] (Figure 1). Similarly, Aghajafari and colleagues concluded that $25(\mathrm{OH}) \mathrm{D}$ levels $<75 \mathrm{nmol} / \mathrm{L}$ were associated with increased odds of GDM (OR 1.49 (95\% confidence interval 1.18 to 1.88 ) based on a meta-analysis of 10 studies [32]. However, the quality of these meta-analyses is limited by the observational nature of the included studies, the mixing of diverse study populations from various regions, and the different laboratory techniques and timing of measurement of serum $25(\mathrm{OH}) \mathrm{D}$ level. 
However, of greatest concern is the inability to understand how important confounding variables such as race/ethnicity and adiposity might change the effect estimates.

\section{Trials of Vitamin D in Pregnancy}

While there are several ongoing randomized controlled trials (RCT) of vitamin D supplementation in pregnancy [33], few are targeted at treatment of GDM and none is testing prevention of GDM. In our search of the scientific literature, we located just one trial of vitamin D supplementation and GDM. Rudnicki and Mølsted-Pedersen enrolled 12 nulliparous women in Denmark with abnormal glucose tolerance tests, defined as two or more serum glucose measurements $3 \mathrm{SD}$ above the mean [34]. Women underwent a fasting, oral glucose tolerance (OGTT) with $75 \mathrm{~g}$ of glucose. Each subject continued their normal diets over the following two days after which they underwent a second OGTT. Two hours before this second test, subjects received $2 \mu \mathrm{g} / \mathrm{m}^{2}$ of 1,25 -dihydroxyvitamin $\mathrm{D}_{3}$ (Etalpha) intravenously. For the next two weeks they received a daily dose of $0.25 \mu \mathrm{g}$ Etalpha orally and then underwent a third OGTT. Glucose and insulin measurement were obtained before each OGTT and at 30 minute increments for 3 hours afterward. Only IV (not oral) vitamin D administration lowered serum glucose levels compared to baseline, from 5.6 to $4.8 \mathrm{mmol} / \mathrm{L}$ $(\mathrm{P}<0.01)$. Post OGTT insulin levels were significantly lower $(\mathrm{P}<0.05)$ (compared to baseline) after IV vitamin D administration. With oral administration insulin levels were lower but this difference did not reach statistical significance $(\mathrm{P}=0.13)$. Nonetheless, lower insulin levels suggest that the mechanism of improved glucose tolerance was not from increased insulin production but potentially increased insulin sensitivity.

In our searches, we did note one other RCT of likely relevance to the relationship between vitamin D and GDM. Soheilykhah and colleagues recently published a data on various vitamin $\mathrm{D}$ supplementation regimens and measures of insulin resistance in pregnant, nondiabetic women, [35]. The investigators enrolled 120 pregnant, non-insulin-requiring women in Iran during the women's first trimester of pregnancy and obtained fasting blood glucose, insulin levels and 25(OH)D levels. Women were then randomized to one of three Vitamin D groups: 200 IU daily, 2000 IU daily, or 4000 IU daily. At the end of pregnancy, fasting blood samples were again obtained for blood glucose, insulin and 25(OH)D levels. The authors demonstrated dose-response relationships for two of the three measures. Specifically, in the highest supplemented groups, 25(OH)D levels rose the most and insulin levels rose the least (Table 1). Fasting glucose levels in these non-diabetic women were unchanged. The HOMA-IR (the product of glucose and insulin levels and a measurement of insulin resistance) was lower (better) in the highest supplemented group compared to lowest supplemented group ( 2.2 vs. 3.0, respectively). Calcium levels were similar across treatment groups. Although this study included non-diabetic women and thus may not be generalizable to women with GDM, it provides compelling evidence that supplementation with high doses of vitamin D may improve insulin sensitivity.

\section{Challenges to Analyses of Vitamin D and Health}

Studying vitamin D status and health outcomes attracts investigators from a variety of fields because of the widespread actions of vitamin $\mathrm{D}$, the ubiquity of vitamin $\mathrm{D}$ receptors in the human body, and the fascinating epidemiology of vitamin D deficiency. Causal or not, vitamin D status tracks with several risk factors for poor health outcomes. Importantly, in the United States, low 25(OH)D levels are most prevalent in African Americans [36] and overweight/obese individuals [2, 18, 19]. Large disparities in health outcomes persist between black and white Americans and much interest persists in investigating the potential contribution of vitamin D deficiency to health outcomes that differ by race, including birth outcomes [37]. However, in the case of GDM, disparities are not exclusive to black-white 
differences. Asian and Hispanic women have higher rates of GDM compared to white women [12], with smaller differences in vitamin D status compared to black-white vitamin $\mathrm{D}$ disparities [22]. In contrast, obesity is clearly both associated with vitamin D deficiency and with GDM $[16,17]$. Whether suboptimal vitamin D status causes an increased risk in GDM remains unknown and may be difficult to tease out from risk factors that may act as effect measure modifiers and confounders. For example, whether vitamin D deficiency affects an obese woman's risk of GDM differently than a lean woman's risk of GDM remains unknown. Based on the very limited human trials of vitamin D supplementation during pregnancy, the vitamin D-induced increase in insulin sensitivity lends biologic plausibility to a threshold phenomenon. Further, regardless of whether optimal vitamin D status can prevent GDM, the limited trial data suggest that exploring an adjunctive role of vitamin D supplementation for women with established GDM may be fruitful. While additional observational studies on the topic are likely, the field needs well-designed RCTs to answer this and other important questions about the relationship between vitamin $\mathrm{D}$ and GDM.

\section{Conclusion}

The current body of literature examining the association between vitamin D status and GDM is largely comprised of conflicting observational studies. This work recently culminated in two well-done meta-analyses that presented evidence of a modest association between low 25(OH)D level and increased odds of GDM. However, whether vitamin D deficiency contributes to the pathophysiology of the development of GDM remains unknown. To our knowledge, no large randomized trial of various vitamin $\mathrm{D}$ doses in women either at high risk for developing GDM or with prior GDM has been published. The only RCTs available are promising but far from definitive, and RCTs are critical to demonstrating a protective effect of optimal vitamin D status with respect to the development or management of GDM.

Current recommendations from the American College of Obstetrics and Gynecology (ACOG) do not recommend routine screening for $25(\mathrm{OH}) \mathrm{D}$ level in pregnancy nor vitamin D supplementation beyond what is contained in a prenatal vitamin [38]. However, ACOG suggests that for women at high risk for vitamin D deficiency, screening may considered and if women are found to be deficient then supplementation with 1000-2000 IU is reasonable. ACOG does not specifically list GDM as being associated with vitamin D deficiency, but the scientific literature suggests that women with GDM are at higher risk than normoglycemic women of low 25(OH)D levels even if the causality of the vitamin D deficiency - GDM association is not yet clear. The next challenge for clinician researchers is to determining whether optimal vitamin D status can prevent GDM and whether vitamin D supplementation for diabetic women with vitamin D deficiency improves glucose tolerance, thus improving perinatal outcomes for mothers and their infants.

\section{Acknowledgments}

Dr. Burris's work is supported by the Klarman Family Foundation Scholars Program at Beth Israel Deaconess Medical Center and by NIH/NIEHS K23 ES022242.

\section{Abbreviations}

$\begin{array}{ll}\text { 25(OH)D } & \text { 25-hydroxyvitamin D } \\ \text { GDM } & \text { gestational diabetes mellitus } \\ \text { DBP } & \text { vitamin D binding protein }\end{array}$




$\begin{array}{ll}\text { VDR } & \text { vitamin D receptor } \\ \text { VDRE } & \text { vitamin D responsive elements } \\ \text { OGTT } & \text { oral glucose tolerance test } \\ \text { RCT } & \text { randomized controlled trial } \\ \text { HOMA-IR } & \text { homeostatic model assessment of insulin resistance } \\ \text { ACOG } & \text { American College of Obstetricians and Gynecologists }\end{array}$

\section{References}

Papers of particular interest, published recently, have been highlighted as:

- Of importance

•• Of major importance

1. Ross, AC. Dietary reference intakes for calcium and vitamin D. Washington, DC: National Academies Press; 2011. Institute of Medicine (U. S.). Committee to Review Dietary Reference Intakes for Vitamin D and Calcium.

2. Holick MF. Vitamin D deficiency. N Engl J Med. 2007; 357:266-281. [PubMed: 17634462]

3. Hypponen E, Laara E, Reunanen A, et al. Intake of vitamin D and risk of type 1 diabetes: a birthcohort study. Lancet. 2001; 358:1500-1503. [PubMed: 11705562]

4. Bourlon PM, Billaudel B, Faure-Dussert A. Influence of vitamin D3 deficiency and 1,25 dihydroxyvitamin D3 on de novo insulin biosynthesis in the islets of the rat endocrine pancreas. J Endocrinol. 1999; 160:87-95. [PubMed: 9854180]

5. Cade C, Norman AW. Vitamin D3 improves impaired glucose tolerance and insulin secretion in the vitamin D-deficient rat in vivo. Endocrinology. 1986; 119:84-90. [PubMed: 3013599]

6. Pittas AG, Lau J, Hu FB, et al. The role of vitamin D and calcium in type 2 diabetes. A systematic review and meta-analysis. J Clin Endocrinol Metab. 2007; 92:2017-2029. [PubMed: 17389701]

7. Ferrara A, Kahn HS, Quesenberry CP, et al. An increase in the incidence of gestational diabetes mellitus: Northern California, 1991-2000. Obstet Gynecol. 2004; 103:526-533. [PubMed: 14990417]

8. Committee opinion, no. 504: screening and diagnosis of gestational diabetes mellitus. Obstet Gynecol. 2011; 118:751-753. [PubMed: 21860317]

9. Jovanovic L, Pettitt DJ. Gestational diabetes mellitus. JAMA. 2001; 286:2516-2518. [PubMed: 11722247]

10. Kjos SL, Buchanan TA. Gestational diabetes mellitus. N Engl J Med. 1999; 341:1749-1756. [PubMed: 10580075]

11. Ben-Haroush A, Yogev Y, Hod M. Epidemiology of gestational diabetes mellitus and its association with Type 2 diabetes. Diabet Med. 2004; 21:103-113. [PubMed: 14984444]

12. Bardenheier BH, Elixhauser A, Imperatore G, et al. Variation in prevalence of gestational diabetes mellitus among hospital discharges for obstetric delivery across 23 States in the United States. Diabetes Care. 2013; 36:1209-1214. [PubMed: 23248195]

13. Harlev A, Wiznitzer A. New insights on glucose pathophysiology in gestational diabetes and insulin resistance. Curr Diab Rep. 2010; 10:242-247. [PubMed: 20425589]

14. Dror DK. Vitamin D status during pregnancy: maternal, fetal, and postnatal outcomes. Curr Opin Obstet Gynecol. 2011; 23:422-426. [PubMed: 21986726]

15. Ozfirat Z, Chowdhury TA. Vitamin D deficiency and type 2 diabetes. Postgrad Med J. 2010; 86:18-25. quiz 4. [PubMed: 20065337]

16. Chu SY, Callaghan WM, Kim SY, et al. Maternal obesity and risk of gestational diabetes mellitus. Diabetes Care. 2007; 30:2070-2076. [PubMed: 17416786] 
17. Solomon CG, Willett WC, Carey VJ, et al. A prospective study of pregravid determinants of gestational diabetes mellitus. JAMA. 1997; 278:1078-1083. [PubMed: 9315766]

18. Parikh SJ, Edelman M, Uwaifo GI, et al. The relationship between obesity and serum 1,25dihydroxy vitamin D concentrations in healthy adults. J Clin Endocrinol Metab. 2004; 89:11961199. [PubMed: 15001609]

19. Cheng S, Massaro JM, Fox CS, et al. Adiposity, cardiometabolic risk, and vitamin D status: the Framingham Heart Study. Diabetes. 2009; 59:242-248. [PubMed: 19833894]

20. Soheilykhah S, Mojibian M, Rashidi M, et al. Maternal vitamin D status in gestational diabetes mellitus. Nutr Clin Pract. 2010; 25:524-527. [PubMed: 20962313]

21. Bischoff-Ferrari HA, Giovannucci E, Willett WC, et al. Estimation of optimal serum concentrations of 25-hydroxyvitamin D for multiple health outcomes. Am J Clin Nutr. 2006; 84:18-28. [PubMed: 16825677]

22. Ginde AA, Sullivan AF, Mansbach JM, et al. Vitamin D insufficiency in pregnant and nonpregnant women of childbearing age in the United States. Am J Obstet Gynecol. 2010; 202:436. e1-8. [PubMed: 20060512]

23. Holmes VA, Barnes MS, Alexander HD, et al. Vitamin D deficiency and insufficiency in pregnant women: a longitudinal study. Br J Nutr. 2009; 102:876-881. [PubMed: 19331703]

24. van den Ouweland JM, Beijers AM, Demacker PN, et al. Measurement of 25-OH-vitamin D in human serum using liquid chromatography tandem-mass spectrometry with comparison to radioimmunoassay and automated immunoassay. J Chromatogr B Analyt Technol Biomed Life Sci. 2010; 878:1163-1168.

25. Clifton-Bligh RJ, McElduff P, McElduff A. Maternal vitamin D deficiency, ethnicity and gestational diabetes. Diabet Med. 2008; 25:678-684. [PubMed: 18544105]

26. Hossein-Nezhad A, Maghbooli Z, Vassigh AR, et al. Prevalence of gestational diabetes mellitus and pregnancy outcomes in Iranian women. Taiwan J Obstet Gynecol. 2007; 46:236-241. [PubMed: 17962102]

27. Zhang C, Qiu C, Hu FB, et al. Maternal plasma 25-hydroxyvitamin D concentrations and the risk for gestational diabetes mellitus. PLoS One. 2008; 3:e3753. [PubMed: 19015731]

28. Farrant HJ, Krishnaveni GV, Hill JC, et al. Vitamin D insufficiency is common in Indian mothers but is not associated with gestational diabetes or variation in newborn size. Eur J Clin Nutr. 2009; 63:646-652. [PubMed: 18285809]

29. Makgoba M, Nelson SM, Savvidou M, et al. First-trimester circulating 25-hydroxyvitamin d levels and development of gestational diabetes mellitus. Diabetes Care. 2011; 34:1091-1093. [PubMed: 21454797]

30. Burris HH, Rifas-Shiman SL, Kleinman K, et al. Vitamin D deficiency in pregnancy and gestational diabetes mellitus. Am J Obstet Gynecol. 2012

31. Wei SQ, Qi HP, Luo ZC, et al. Maternal vitamin D status and adverse pregnancy outcomes: a systematic review and meta-analysis. J Matern Fetal Neonatal Med. 2013 Recently published systematic review of $25(\mathrm{OH}) \mathrm{D}$ levels and pregnancy outcomes including a meta-analysis for GDM. The authors included 12 studies of 5615 participants and found that among women with $25(\mathrm{OH}) \mathrm{D}$ levels $<50 \mathrm{nmol} / \mathrm{L}$ increase in odds of GDM (Figure 1)

32. Aghajafari F, Nagulesapillai T, Ronksley PE, et al. Association between maternal serum 25hydroxyvitamin D level and pregnancy and neonatal outcomes: systematic review and metaanalysis of observational studies. BMJ. 2013; 346:f1169. [PubMed: 23533188] Another recently published systematic review of vitamin D status and pregnancy outcomes including a metaanalysis of 10 studies of $25(\mathrm{OH}) \mathrm{D}$ levels and the risk of GDM. They concluded that women with 25(OH)D. levels $<75 \mathrm{nmol} / \mathrm{L}$ had higher odds of GDM (Figure 1.)

33. ClinicalTrials.gov. [Accessed May 28, 2013] (http://www.clinicaltrials.gov/ct2/results?term= $\% 22$ Vitamin+D\%22+and+\%22pregnancy\%22\&Search=Search).

34. Rudnicki PM, Molsted-Pedersen L. Effect of 1,25-dihydroxycholecalciferol on glucose metabolism in gestational diabetes mellitus. Diabetologia. 1997; 40:40-44. [PubMed: 9028716]

35. Soheilykhah S, Mojibian M, Moghadam MJ, et al. The effect of different doses of vitamin D supplementation on insulin resistance during pregnancy. Gynecol Endocrinol. 2013; 29:396-399. [PubMed: 23350644] This is a recent trial of various doses of vitamin D supplementation with 
measurement of insulin resistance among non-diabetic pregnant women. Findings included similar glucose concentrations across groups, but lower insulin levels among women with higher doses of vitamin D supplementation, suggesting increased insulin sensitivity (Table 1)

36. Nesby-O'Dell S, Scanlon KS, Cogswell ME, et al. Hypovitaminosis D prevalence and determinants among African American and white women of reproductive age: third National Health and Nutrition Examination Survey, 1988-1994. Am J Clin Nutr. 2002; 76:187-192. [PubMed: 12081833]

37. Bodnar LM, Simhan HN. Vitamin D may be a link to black-white disparities in adverse birth outcomes. Obstet Gynecol Surv. 2010; 65:273-284. [PubMed: 20403218]

38. ACOG Comittee on Obstetric Practice. ACOG Committee Opinion No. 495: Vitamin D: Screening and supplementation during pregnancy. Obstet Gynecol. 2011; 118:197-198. [PubMed: 21691184]

39. Wei SQ, Qi HP, Luo ZC, et al. Maternal vitamin D status and adverse pregnancy outcomes: a systematic review and meta-analysis. J Matern Fetal Neonatal Med. 2013; 26:889-899. [PubMed: 23311886] 


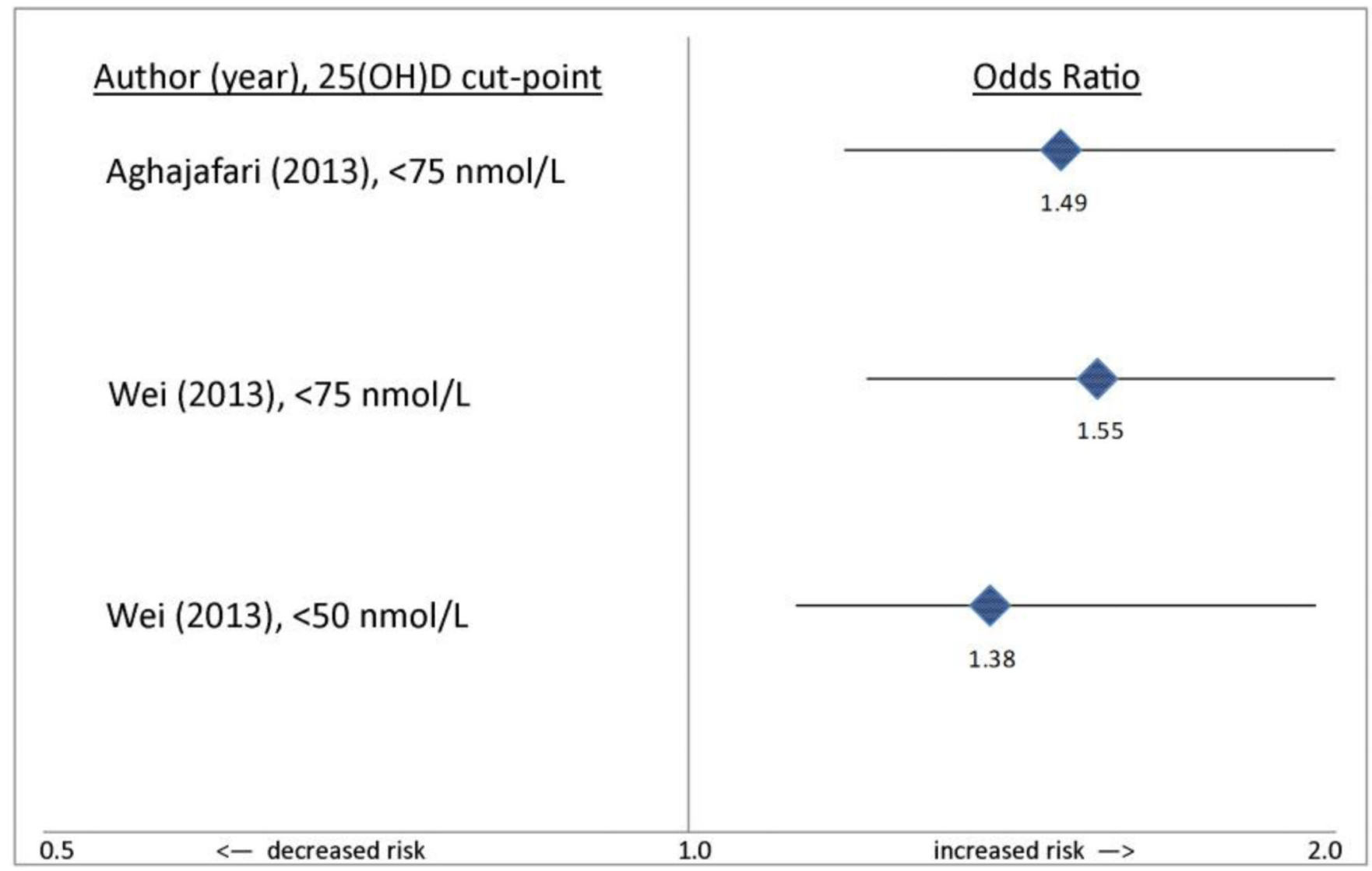

Figure 1.

Associations between 25-hydroxyvitamin D levels and odds of gestational diabetes mellitus, results from two systematic reviews/meta-analyses [31, 32]. (Created from odds ratio (95\% confidence interval) published by Wei and colleagues [39] and Aghajafari and colleagues[32]; 25(OH)D denotes 25-hydroxyvitamin D.) 


\section{Table 1}

First trimester and end of pregnancy plasma markers of vitamin D status and insulin resistance before and after daily oral vitamin D supplementation of 200 IU, 2000 IU, and 4000 IU

\begin{tabular}{|c|c|c|c|c|}
\hline & $\begin{array}{c}200 \text { IU/day } \\
\underline{n=35}\end{array}$ & $\begin{array}{c}2000 \text { IU/day } \\
\underline{n=38}\end{array}$ & $\begin{array}{c}4000 \mathrm{IU} / \mathrm{day} \\
\underline{\mathrm{n}=40}\end{array}$ & $\underline{P}$ \\
\hline \multicolumn{5}{|c|}{$25(\mathrm{OH}) \mathrm{D}$ level ng/ml } \\
\hline Before & 8.3 & 7.3 & 7.3 & 0.95 \\
\hline After & 17.7 & 27.2 & 34.1 & 0.001 \\
\hline \multicolumn{5}{|c|}{ Insulin (IU/ml) } \\
\hline Before & 8.3 & 7.4 & 8.0 & 0.52 \\
\hline After & 15.3 & 12.2 & 11.6 & 0.009 \\
\hline \multicolumn{5}{|c|}{ HOMA-IR } \\
\hline Before & 1.6 & 1.4 & 1.5 & 0.74 \\
\hline After & 3.0 & 2.4 & 2.2 & 0.01 \\
\hline \multicolumn{5}{|c|}{ Fasting blood sugar (mg/dl) } \\
\hline Before & 76.6 & 78.8 & 78.0 & 0.23 \\
\hline After & 77.6 & 79.0 & 76.0 & 0.04 \\
\hline \multicolumn{5}{|l|}{ Calcium } \\
\hline Before & 9.7 & 9.7 & 9.6 & 0.28 \\
\hline After & 9.4 & 9.5 & 9.2 & 0.04 \\
\hline
\end{tabular}

Results from a trial in Iran of 113 women by Soheilykhah and colleagues [35].

Abbreviations: 25(OH)D, 25-hydroxyvitamin D; HOMA-IR, homeostatic model assessment of insulin resistance. 